\title{
INCIDENCIA DE INFECCIÓN DEL TRACTO URINARIO ASOCIADO A CATÉTER VESICAL
}

\author{
INCIDENCE OF INFECTION OF THE URINARY TRACT ASSOCIATED WITH A \\ VESICAL CATHETER
}

\author{
Carlos Proaño Larrea \\ Hospital General de Especialidades de las Fuerzas Armadas del Ecuador \\ Email: umsmwork74@gmail.com \\ https://doi.org/10.33789/talentos.8.1.145
}

\begin{abstract}
Resumen: La infección del tracto urinario constituye uno de los problemas de salud que con mayor frecuencia se presentan en la práctica médica diaria; uno de sus posibles factores desencadenantes es el uso de catéter vesical. El objetivo de esta investigación fue determinar la incidencia de infección del tracto urinario asociado al uso de catéter vesical. Para esto se realizó una investigación básica, descriptiva y explicativa que incluyó a 377 pacientes con infección intrahospitalaria del tracto urinario. Los principales resultados incluyen promedio de edad de 57,63 años, predominio de pacientes femeninas (63,92\%) y con comorbilidades asociadas $(54,30 \%)$. La incidencia de infección del tracto urinario en pacientes con cateterismo vesical fue de 21,24\%. Del total de pacientes en el cual se confirmó el proceso infeccioso el $23,61 \%$ utilizó catéter vesical y predominaron los pacientes masculinos (36,03\%) y con comorbilidades asociadas (32,74\%). En pacientes con catéter vesical y diagnóstico de lupus eritematoso sistémico $(61,11 \%)$, diabetes mellitus $(53,33 \%)$ y artritis reumatoide $(43,75 \%)$ fueron en los que en mayor porciento de infección del tracto urinario se identificó. Se concluye que existió una elevada incidencia de infección del tracto urinario en pacientes con catéter vesical. La presencia de comorbilidades y el sexo femenino fueron factores que se identificaron con mayor frecuencia en la totalidad de pacientes con infección del tracto urinario; sin embargo su asociación a cateterismo vesical fue más frecuente en pacientes masculinos.
\end{abstract}

Palabras claves: Artritis reumatoide; Catéter vesical; Diabetes mellitus; Infección tracto urinario; Lupus Eritematoso Sistémico.

Recibido: 23 de octubre de 2020

Aceptado: 06 de mayo de 2021

Publicado como artículo científico en la Revista de Investigación Talentos VIII (1), 84-92 
Abstract: Urinary tract infection is one of the health problems that most frequently occurs in daily medical practice; one of its possible triggers is the use of a bladder catheter. The objective of this research was to determine the incidence of urinary tract infection associated with the use of a bladder catheter. For this, a basic, descriptive and explanatory research was carried out that included 377 patients with intrahospital urinary tract infection. The main results include an average age of 57.63 years, a predominance of female patients (63.92\%) and with associated comorbidities $(54.30 \%)$. The incidence of urinary tract infection in patients with bladder catheterization was $21.24 \%$. Of the total number of patients in whom the infectious process was confirmed, $23.61 \%$ used a bladder catheter and male patients (36.03\%) and with associated comorbidities (32.74\%) predominated. In patients with a bladder catheter and a diagnosis of systemic lupus erythematosus (61.11\%), diabetes mellitus (53.33\%) and rheumatoid arthritis $(43.75 \%)$ were those with a higher percentage of urinary tract infection. It is concluded that there was a high incidence of urinary tract infection in patients with a bladder catheter. The presence of comorbidities and female sex were factors that were identified more frequently in all patients with urinary tract infection; however, its association with bladder catheterization was more frequent in male patients.

Key words: Rheumatoid arthritis; Bladder catheter; Mellitus diabetes; Urinary tract infection; Systemic lupus erythematosus.

\section{INTRODUCCIÓN}

Las infecciones del tracto urinario (ITU) son conceptualizados como los procesos inflamatorios relacionados con la invasión y multiplicación de microorganismos patógenos en cualquier nivel del tracto urinario. Se describe como formas clínicas de presentación de la enfermedad la uretritis, cistitis, pielonefritis y, en casos complicados, sepsis de origen urinario (CDC, 2016). Son considerados como las enfermedades infecciosas que con mayor frecuencia se presentan en el ambiente hospitalario. Se reporta que la ITU representra entre el $25 \%$ y el $45 \%$ de las infecciones nosocomiales (Lossa, et al., 2008).

Se describe que el principal factor asociado a la ITU hospitalaria es el uso del catéter vesical. Se considera como infección nosocomial cuando la enfermedad se presenta en el transcurso de los cinco días posteriores a la colocación de la sonda de Foley (Calderón Jaimes, 2013). Se describe que el $80 \%$ de los pacientes que presentan complicaciones por ITU hospitalaria se encuentran sondeados de forma permanente (Reyes, et al, 2014).

Se describen múltiples factores asociados a la peresencia de ITU en pacientes que utilizan sonda vesical. Destacan el tiempo de utilización de la sonda, las complicaciones durante la colocación, el manejo del catéter, la presencia de comorbilidades asociadas, el embarazo, las alteraciones anátomo funcionales del tracto urinario y la susceptibilidad del huésped según su edad y sexo. (Fariñas Álvarez, Teira Cobo, \& Rodríguez Cundín, 2010).

La gran mayoría de las ITU son consideradas como no complicadas, siendo mayormente diagnosticadas en pacientes jóvenes y del sexo femenino. Por lo general no requieren pruebas diagnósticas complementarias; se tratan ambulatoriamente y de forma segura con antibióticos utilizados por vía oral. Sin embargo, si se sospecha de una infección asociada a cateterismo vesical es necesario indicar exámenes complementarios para corroborarla, valorar la posible resistencia antibiótica e identificar el microorganismo causante de dicha infección. Lo que facilita la prescripción de fármacos (antibióticos) específicos. (de Cueto et al., 2017). Se estima que la prevalencia de infecciones asociadas a la asistencia sanitaria ronda el 5\% de los pacientes hospitalizados. De estos, la infección del tracto urinario constituye la causa principal y se presenta en el $1,5 \%$ de 
los pacientes hospitalizados. (Carrouget, et al., 2017).

El cateterismo vesical es una medida que se utiliza con frecuencia en el ámbito hospitalario. Se prescribe su utilización en pacientes con estados de salud delicados y en los que son sometidos a distintos procederes quirúrgicos entre otros (Empaire, et al, 2017). Su manejo debe ser seguro y se debe monitorear las condiciones del catéter o sonda vesical frecuentemente. El cateterismo vesical es considerado como una de las principales causas de ITU hospitalarias. (Carrouget, et al., 2017)

En Ecuador, son escasos los estudios orientados hacia la identificación de las causas y factores de sepsis nosocomial hospitalaria; mucho más aún en el tema relacionado con la utilización de catéter vesical y sus posibles complicaciones infecciosas. Es por esto, que teniendo en cuenta los reportes internacionales que muestran una elevada incidencia de ITU en pacientes hospitalizados; se decide realizar esta investigación con el objetivo de determinar la incidencia de ITU asociado a catéter vesical en Hospital de Especialidades de las Fuerzas Armadas del Ecuador (HE1) durante el periodo comprendido entre los meses de enero y diciembre del 2017.

\section{METODOLOGÍA}

Se realizó una investigación básica, no experimental, con un diseño descriptivo y explicativo. El enfoque fue mixto ya que incluyó elementos cualitativos y cuantitativos. El universo estuvo constituido por la totalidad de los pacientes con ITU intrahospitalaria durante el periodo de estudio (403). Para realizar el cálculo del tamaño de la muestra se utilizó la forma de cálculo muestral para poblaciones conocidas. Después de realizar los cálculos matemáticos se definió el total de la muestra en 377 pacientes. Se utilizó el método de muestreo aleatorio simple para conformar la muestra y cada uno de los pacientes incluidos en el estudio cumplió con los criterios de inclusión y exclusión definidos para la investigación.
Criterios de inclusión

- Pacientes adultos (mayores de 18 años), hospitalizados en el periodo enero diciembre 2017 en el Hospital HE-1 en los servicios de medicina Interna, Cirugía general y Urología y que se confirmó el diagnóstico de ITU intra hospitalaria, mediante la realización de urocultivo y antibiograma.

- Pacientes en cuyas historias clínicas contaban todos los elementos necesarios para dar cumplimiento al objetivo del estudio.

Criterios de exclusión

- Pacientes embarazadas.

- Pacientes con diagnóstico de ITU confirmado pero hospitalizados en otros servicios.

- Pacientes con diagnóstico presuntivo de ITU en los cuales no se realizó urocultivo y/o antibiograma.

- Pacientes cuyas historias clínicas no contenían toda la información necesaria para dar cumplimiento al objetivo de la investigación.

Para la realización de la investigación se tuvieron en cuenta las siguientes variables y subvariables. La variable características generales con las subvariables edad, sexo, presencia de comorbilidades asociadas y tipo de comorbilidades asociadas. La variable cateterismo vesical mostró el número $\mathrm{y}$ porciento de pacientes con ITU según el uso o no de catéter vesical.

Se utilizó, como técnica de investigación, la revisión documental que consistió en la revisión de la historia clínica de cada paciente incluido en la investigación. Mediante esta técnica se pudo obtener toda la información relacionada con las variables y subvariables definidas para el estudio. Toda la información recopilada fue homogenizada en un modelo de recolección de información creado 
específicamente para la investigación.

El procesamiento de la información se realizó de forma automatizada con la ayuda del programa estadísticos SPSS en su versión 20,5 para Windows. Se determinaron medidas de tendencia central y de dispersión para las variables cuantitativas y frecuencias absolutas y porcentajes para las variables cualitativas. Se definió el nivel de confianza en el 95\%, el margen de error en el 5\% y la significación estadística en una p menor o igual de 0,05 . Todos los resultados fueron expresados en forma de tablas estadísticas para facilitar su comprensión.

Las normas éticas incluidas en la investigación estuvieron en concordancia con las normas y procedimientos establecidos en la declaración de Helsinki II para la realización de investigaciones en seres humanos. Los datos obtenidos fueron utilizados únicamente con fines investigativos y no se utilizaron datos de identificación personal durante el desarrollo de la investigación. La base de datos, con toda la información recopilada, fue destruida al terminar el informe final de la investigación.

\section{RESULTADOS Y DISCUSIÓN}

Tabla 1. Distribución de pacientes con ITU según características generales

\begin{tabular}{ll}
\hline Características generales & $\begin{array}{l}\text { Muestra total } \mathbf{n}=\mathbf{3 7 7} \\
\text { Frecuencia (por ciento) }\end{array}$ \\
\hline $\begin{array}{l}\text { Promedio de edad (años) } \\
\text { Sexo }\end{array}$ & $57,63 * \mathrm{DE} 11,37$ \\
Masculino & $136(36,08)$ \\
Femenino & $241(63,92)$ \\
Comorbilidades asociadas & \\
Presencia & $223(54,30)$ \\
Ausencia & $154(45,70)$ \\
Tipo de comorbilidades $\mathbf{n}=\mathbf{2 2 3}$ & \\
Diabetes mellitus & $105(47,08)$ \\
Hipotiroidismo & $53(23,77)$ \\
Hipertensión arterial & $31(13,90)$ \\
Artritis reumatoide & $16(7,17)$ \\
Lupus eritematoso sistémico & $18(8,07)$ \\
\hline
\end{tabular}

Fuente: cuestionario de investigación

Se observa en la tabla 1 que existió un promedio de edad de 57,63 años con DE de 11,47 años. Existió un predominio de pacientes femeninas con ITU $(63,92 \%)$ en comparación con los pacientes masculinos con ITU $(36,08 \%)$. Al analizar la presencia o ausencia de comorbilidades asociadas se obtuvo que el $54,30 \%$ de los pacientes con ITU presentaban al menos una comorbilidad asociada. Dentro de las comorbilidades asociadas la diabetes mellitus fue la de mayor frecuencia de reporte $(47,08 \%)$, le siguieron el hipotiroidismo $(23,77 \%)$ y la hipertensión arterial $(13,90 \%)$. Dentro de los pacientes con ITU el 8,07\% presentaban diagnóstico de lupus eritematoso sistémico y el 7,17\% de artritis reumatoide. 
Tabla 2. Distribución de pacientes con ITU y uso de catéter vesical

\begin{tabular}{|c|c|c|}
\hline \multirow[t]{2}{*}{ Uso de catéter vesical } & \multicolumn{2}{|c|}{ Muestra total $n=377$ paciente } \\
\hline & Frecuencia & Porciento \\
\hline Uso de catéter vesical & 89 & 23,61 \\
\hline No uso de catéter vesical & 288 & 76,39 \\
\hline Total & 377 & 100 \\
\hline
\end{tabular}

Se observa en la tabla 2 que el $23,61 \%$ de los pacientes que desarrollaron ITU estuvieron con catéter vesical.

E1 76,39\% de los pacientes que desarrollaron
ITU no presentaban en el momento del diagnóstico catéter vesical.

Tabla 3. Distribución de pacientes según presencia de ITU secundaria al uso de catéter vesical y características generales.

\begin{tabular}{|c|c|c|c|c|c|}
\hline \multirow{3}{*}{ Características generales } & \multicolumn{5}{|c|}{ Muestra total $n=377$ pacientes con ITU } \\
\hline & \multicolumn{2}{|c|}{ 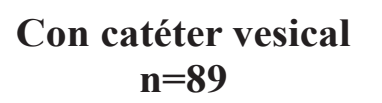 } & \multicolumn{2}{|c|}{ Sin catéter vesical $n=288$} & \multirow[b]{2}{*}{$\mathbf{p}$} \\
\hline & $\begin{array}{l}\text { Frecuen- } \\
\text { cia }\end{array}$ & $\begin{array}{l}\text { Porcien- } \\
\text { to }\end{array}$ & Frecuencia & Porciento & \\
\hline \multicolumn{6}{|l|}{ Sexo } \\
\hline Masculino & 49 & 36,03 & 87 & 63,97 & ---- \\
\hline Femenino & 40 & 16,60 & 161 & 83,40 & ---- \\
\hline \multicolumn{6}{|l|}{ Comorbilidades asociadas } \\
\hline Presencia & 73 & 32,74 & 150 & 67,26 & ---- \\
\hline Ausencia & 16 & 10,39 & 138 & 89,61 & ---- \\
\hline \multicolumn{6}{|c|}{ Tipo de comorbilidades asociadas } \\
\hline Diabetes mellitus & 56 & 53,33 & 49 & 46,67 & 0,047 \\
\hline Hipotiroidismo & 10 & 18,87 & 43 & 81,13 & 0,082 \\
\hline Hipertensión arterial & 5 & 16,13 & 26 & 83,87 & 0,084 \\
\hline Artritis reumatoide & 7 & 43,75 & 9 & 56,25 & 0,057 \\
\hline Lupus eritematoso sistémico & 11 & 61,11 & 7 & 38,89 & 0,039 \\
\hline
\end{tabular}

Fuente: cuestionario de investigación

Existió un mayor por ciento de pacientes masculinos con catétervesicaly ITU $(36,03 \%)$, en relación a las pacientes femeninas con ITU y cateterismo vesical $(16,60 \%)$. Los pacientes con comorbilidades asociadas $\mathrm{y}$ uso de catéter vesical presentaron un mayor por ciento de ITU $(32,74 \%)$ que los que no refirieron presentar alguna comorbilidad asociada $(10,39 \%)$. Dentro del grupo de pacientes con comorbilidades asociadas y diagnóstico de ITU destacan el 61,11\% de los pacientes con lupus eritematoso, el 53,33\% de 
pacientes con diabetes mellitus y el 43,75\% de pacientes con artritis reumatoide. Solo el $18,87 \%$ de los pacientes con hipotiroidismo utilizaron catéter vesical y el $16,13 \%$ de los casos hipertensos (tabla 3).

Tabla 4. Distribución de pacientes según uso de catéter vesical y presencia de ITU.

\begin{tabular}{|c|c|c|}
\hline \multirow{2}{*}{$\begin{array}{l}\text { Presencia } \\
\text { de ITU }\end{array}$} & \multicolumn{2}{|c|}{$\begin{array}{l}\text { Muestra total de pacientes } \\
\text { con catéter vesical } n=419\end{array}$} \\
\hline & Frecuencia & Porciento \\
\hline Con ITU & 89 & 21,24 \\
\hline Sin ITU & 330 & 78,76 \\
\hline Total & 419 & 100,0 \\
\hline
\end{tabular}

Fuente: cuestionario de investigación

Se observa en la tabla 4 que del total de pacientes identificados que utilizaron catéter vesical (419) el 21,24\% presentaron ITU. Un total de 330 pacientes que utilizaron catéter vesical no presentaron ITU $(78,76 \%)$. La incidencia de ITU en pacientes con catéter vesical fue del $21,24 \%$

\section{DISCUSIÓN}

Los procesos inflamatorios intrahospitalarios son considerados como de alto riesgo por el fenómeno de resistencia antimicrobiana que puede estarlos acompañando (Montenegro Díaz, et al., 2016). Se describe dentro de las infecciones nosocomiales o intrahospitalarias las ITU como una de las de mayor frecuencia de presentación y estas se asocian frecuentemente al uso de catéter vesical (Jiménez Bermúdez, Carballo Solís, \& Chacón Jiménez, 2017).

En la presente investigación se obtuvo un promedio de edad de pacientes con ITU superior a los 55 años. Este resultado concuerda con lo reportado por Zúniga Moya y otros (2016) quienes reportan un predominio de la ITU en pacientes mayores de 50 años. Una posible explicación a este resultado puede estar condicionado por el proceso degenerativo que acompaña al envejecimiento en el cual se reporta que existe una disminución considerable de la funcionalidad del sistema inmune (Solis Cartas, \& Calvopiña Bejarano, 2018).

El predominio de pacientes femeninas con ITU ha sido ampliamente reportado (Méndez Chacón, et al, 2017). Se describen entre otras causas la cercanía que existe entre la uretra femenina, el entroitus vaginal y el ano. Esta cercanía permite la migración de determinados gérmenes como la Escherichia Coli desde los márgenes del ano hasta la desembocadura de la uretra, pudiendo colonizar el sistema urinario por vía retrógrada. También puede existir migración de gérmenes desde el entroitus vaginal hacia la uretra en caso de existir vaginosis bacteriana $\mathrm{u}$ otro proceso infeccioso vaginal (Lossa, et al, 2008).

También se describe las características anatómicas de la uretra femenina como un factor predisponente de ITU en el sexo femenino (Calle Núñez, et al, 2017). Las féminas, al tener una uretra más corta, sin estrecheces ni ensanchamientos, están condicionadas a un trayecto más favorable para los gérmenes lo que genera mayor facilidad para colonizar el tracto urinario y aparecer ITU (Montenegro Díaz, et al, 2016).

Un elemento adicional que pudiera estar relacionado con el aumento de la incidencia de ITU en el sexo femenino y en edades superiores a los 50 años es el hecho relacionado con el periodo menopáusico. En este se produce una disminución brusca de la producción de estrógenos y progesterona los que tiene acción inmunomoduladora (Solis Cartas, Calvopiña Bejarano, \& Valdés González, 2019). La disminución del estado inmunomodulador genera una disminución de la secreción de inmunoglobulina A que limita la acción bacteriostática y posibilita la colonización bacteriana de la vía urinaria (Andrade, \& Fernandes, 2016).

La presencia de comorbilidad y en especial de lupus eritematoso, diabetes mellitus y artritis reumatoide fueron los elementos más destacados en torno a la presencia de ITU en los pacientes investigados. Se describe que en las enfermedades reumáticas, por su 
comportamiento sistémico, son propensas a presentar cuadros infecciosos que afectan el trato urinario, especialmente en el lupus eritematosos sistémico, donde la toma renal es muy frecuente e incluye, entre otros, los procesos infecciosos (Sousa, et al., 2017). En el caso de la diabetes mellitus se reporta que el aumento de la glucemia favorece el crecimiento bacteriano. Además de que también existe un componente inmunológico dentro de los mecanismos etiopatogénicos de la enfermedad (Purvis, et al, 2014).

Un resultado destacado de este estudio se relaciona con el predominio de ITU en pacientes masculinos con catéter vesical en relación al sexo femenino. El equipo de investigación no encontró estudios similares que permitieran analizar comparativamente este resultado. Sin embargo, una posible explicación pudiera estar relacionada con la colonización prostática de los gérmenes capaces de producir ITU. A pesar de esto se recoge el antecedente del estudio realizado por Sabir y otros (2017) donde sí se evidenció al género femenino como un factor de riesgo para ITU asociado a catéter vesical, aunque los objetivos y métodos utilizados son diferentes al de esta investigación.

\section{CONCLUSIONES}

Se concluye que existió una incidencia del $21,24 \%$ de presencia de ITU en pacientes con catéter vesical hospitalizados en los servicios de Medicina Interna, Urología y Cirugía General, por lo que se necesita reforzar la atención a los cuidados general en pacientes con catéter vesical para prevenir las ITU. La presencia de comorbilidades y el sexo femenino fueron factores que se identificaron con mayor frecuencia en la totalidad de pacientes con ITU; sin embargo la ITU asociada a cateterismo vesical fue más frecuente en pacientes masculinos.

\section{REFERENCIAS BIBLIOGRÁFICAS}

Andrade, V. L., \& Fernandes, F. A. (2016). Prevención de la infección del tracto urinario asociada al cateterismo: estrategias en la implementación de las directrices internacionales. Revista Latino-Americana de Enfermagem, 24, e2678. Epub March 28, 2016. Recuperado de: https://doi. org/10.1590/1518-8345.0963.2678

Calderón Jaimes, E. (2013). Diagnóstico y tratamiento de las infecciones en vías urinarias: un enfoque multidisciplinario para casos no complicados. Boletín Médico Del Hospital Infantil de Mexico, 70(1), 3-10. Recuperado de http://www. scielo.org. $\mathrm{mx} / \mathrm{scielo}$.php?pid=S1665$11462013000100003 \&$ script $=$ sci $\underline{\operatorname{arttext}}$

Calle Núñez, A., Colqui Campos, K.A., Rivera Estrella, D.A., \& Cieza Zevallos, J.A. (2017). Factores asociados a la presentación de infecciones urinarias por Escherichia coli productoras de betalactamasas de espectro extendido. Revista Medica Herediana, 28(3), 142149. Recuperado de https://dx.doi. org/https://doi.org/10.20453/rmh. v28i3.3180

Carrouget, J., Legeay, C., Poirier, A., Azzouzi, A. R., Zahar, J. R., \& Bigot, P. (2017). Enquête de prévalence sur le sondage vésical dans un centre hospitalo-universitaire. Progres En Urologie, 27(5), 305-311. Recuperado de https://doi.org/10.1016/j. purol.2017.03.006

Centers for Disease Control and Prevention (CDC). (2016). Urinary Tract Infection (Catheter-Associated Urinary Tract Infection [CAUTI] and Non-CatheterAssociated Urinary Tract Infection [UTI]) and Other Urinary System Infection [USI]) Events. In 2016 NHSN Patient Safety Component Manual.

De Cueto, M., Aliaga, L., Alós, J. I., Canut, 
A., LosArcos, I., Martínez, J. A., \& Pigrau, C. (2017). Resumen ejecutivo del diagnóstico y tratamiento de las infecciones del tracto urinario.

Empaire, G.D., Siritt, M.E.G., Rosenthal, V.D., Pérez, F., Ruiz, Y., Díaz, C., \& Orozco, N. (2017). Multicenter prospective study on device-associated infection rates and bacterial resistance in intensive care units of Venezuela: International Nosocomial Infection Control Consortium (INICC) findings. International Health. https://doi. org/10.1093/inthealth/ihw049

Fariñas-Álvarez, C., Teira-Cobo, R., \& Rodríguez-Cundín, P. (2010). Infección asociada a cuidados sanitarios (infección nosocomial). Medicine, 10(49), 3293-3300. Recuperado de https://doi.org/10.1016/ $\underline{\mathrm{S} 0304-5412(10) 70031-7}$

Jiménez Bermúdez, J.P., Carballo Solís, K.D, \& Chacón Jiménez, N.K. (2017). Manejo de infecciones del tracto urinario. Revista Costarricense de Salud Pública, 26(1), 1-10. Retrieved September 19, 2020, from http://www.scielo.sa.cr/scielo. php?script $=$ sci arttext\&pid $=$ S1409$14292017000100001 \& \operatorname{lng}=$ en\&tlng $=$ $\underline{\mathrm{es}}$

Lossa, G.R., Lerena, R.G., Fernández, L.E., Vairetti, J., Díaz, C., Arcidiácono, D., $\&$ Peralta, N. (2008). Prevalencia de infecciones hospitalarias en unidades de cuidados intensivos para adultos en Argentina. Revista Panamericana de Salud Pública. Recuperado de: https:// www.scielosp.org/article/rpsp/2008. v24n5/324-330/

Méndez Chacón, P., Bardales Viguria, F., Ardiles Aniceto, Á., Cervera Álvarez, C., Méndez Chacón Rodriguez, C., \& Vidalón Fernández, A. (2017). Infección del tracto urinario en receptores de trasplante renal. Anales de la Facultad de Medicina, 78(1), 11-16. Recuperado de https://dx.doi. org/10.15381/anales.v78i1.13015

Montenegro Díaz, B., Tafur Ramirez, R., Díaz Vélez, C., \& Fernández Mogollon, J. (2016). Infecciones intrahospitalarias del tracto urinario en servicios críticos de un hospital público de Chiclayo, Perú (20092014). Acta Mdica Peruana, 33(3), 189-194. Recuperado de http://www.scielo.org.pe/scielo. php? script $=$ sci arttext\&pid $=$ S172859172016000300004\&lng=es\&tlng $=$ es

Purvis, S., Gion, T., Kennedy, G., Rees, S., Safdar, N., VanDenBergh, S., \& Weber, J. (2014). Catheter-Associated Urinary Tract Infection. Journal of Nursing Care Quality, 29(2), 141-148. Recuperado de https://doi.org/10.1097/ NCQ.0000000000000037

Reyes, S.V.F., Castellanos, M. del R.P., Rodríguez, Z.N., Veranes, F.N.L., \& Fernández, Z.R. (2014). Infección del tracto urinario por uso del catéter vesical en pacientes ingresados en cuidados intensivos. Medisan, 18(11), 1524-1530. Recuperado de http:// scielo.sld.cu/scielo.php?pid=S1029$\underline{30192014001100006 \& \text { script }=\text { sci }}$ arttext\&tlng=pt

Sabir, N., Ikram, A., Zaman, G., Satti, L., Gardezi, A., Ahmed, A., \& Ahmed, P. (2017). Bacterial biofilm-based catheter-associated urinary tract infections: Causative pathogens and antibiotic resistance. American Journal of Infection Control. Recuperado de https://doi.org/10.1016/j. ajic.2017.05.009

Solis Cartas, U., \& Calvopiña Bejarano, SJ. (2018). Comorbilidades y calidad de vida en Osteoartritis. Revista Cubana de Reumatología, 20(2), e17. Recuperado de https://dx.doi. org/10.5281/zenodo. 1188918 
Solis Cartas, U., Calvopiña Bejarano, S.J., \& Valdés González, E.M. (2019).

Calidad de vida relacionada con la salud en pacientes con osteoartritis del cantón Riobamba. Revista Cubana de Reumatología, 21(1), e55. Recuperado de https://dx.doi.org/10.5281/ zenodo. 2554482

Sousa, Á.F., Queiroz, A.A., Oliveira, L.B., Moura, L. K., Andrade, D., Watanabe, E., \& Moura, M. E. B. (2017). Deaths among the elderly with ICU infections. Revista Brasileira de Enfermagem, 70(4), 733-739. Recuperado de https:// doi.org/10.1590/0034-7167-2016-0611

Zúniga Moya, J.C., Bejarano Cáceres, S., Valenzuela Cervantes, H., Gough Coto, S., Castro Mejía, A., Chinchilla López, C., et al. (2016). Perfil de sensibilidad a los antibióticos de las bacterias en infecciones del tracto urinario. Acta Médica Costarricense, 58(4), 146-154. Recuperado de http://www.scielo.sa.cr/scielo. php?script $=$ sci arttext\&pid $=$ S0001$\underline{60022016000400146 \& \operatorname{lng}=\mathrm{en} \& \operatorname{tln} \mathrm{g}=}$ $\underline{\text { es }}$ 